\title{
Determinación Palinológica del Paleoambiente Holocénico en la parte norte del Estado de Michoacán ${ }^{1}$
}

\author{
MA. SUSANA XELHUANTZI LÓPEZ ${ }^{2}$
}

\begin{abstract}
RESUMEN. La reconstrucción paleoambiental de un sitio de interés arqueológico en la parte norte del estado de Michoacán, se realizó a partir del análisis palinológico de sedimentos de la porción central de la Ciénaga de Zacapu y de la zona de contacto entre las geoformas denominadas "Lomas" y la Ciénaga. Los espectros palinológicos permiten distinguir tres etapas climáticas: de ca. 8100 a ca. 6700 años antes del presente, ambiente templado húmedo o sub-húmedo; de ca. 6700 - ca. 5000 años antes del presente, ambiente templado semi-seco; de ca. 5000 años antes del presente a la época actual, ambiente templado sub-húmedo. Los datos palinológicos sugieren que aproximadamente desde 7000 años antes del presente, el antiguo Lago de Zacapu ha sido un depósito con características de ciénaga, reflejando mayores variaciones en las condiciones de humedad que en la temperatura y sugiriendo que durante los últimos 5000 años, el clima en el área de Zacapu, ha sido templado sub-húmedo.
\end{abstract}

ABSTRACT. The palaeoenvironmental reconstruction of a site of archaeological interest in the Basin of Zacapu, in the north of the state of Michoacan, Mexico, was performed through pollen analysis. From the analysis of sediments of the central part of the swamp of Zacapu and the contact zone "Lomas"-swamp, it is possible to distinguish three climatic stages: from ca. 8100 to ca. $6700 \mathrm{BP}$, temperate-humid or subhumid weather; from ca. 6700 to ca. $5000 \mathrm{BP}$, temperate-semiarid weather; from ca. $5000 \mathrm{BP}$ to the present time. temperate-subhumid weather. This study suggests that since $7000 \mathrm{BP}$, the ancient Lake of Zacapu has been a swamp-like water deposit. The climatic changes which can be infered from this paly nological analysis show more variations in the conditions of humidity than in the thermal conditions. In the last $5000 \mathrm{BP}$, the weather in the area of Zacapu has been almost the same as it is in the present time: temperate subhumid.

\footnotetext{
1 Trabajo que formó parte de un proyecto arqueológico-ambiental desarrollado por el CEMCA y el INAH, con la colaboración del Instituto de Geología de la UNAM.

${ }^{2}$ Subdirección de Servicios Académicos, Instituto Nacional de Antropología e Historia, Moneda 16, Centro, Delegación Cuauhtémoc, 06060, México, D.F.
} norte del Estado de Michoacán. Boletín de la Sociedad Botánica de México 54: 251-265. 
La zona que comprende la Cuenca de Zacapu y áreas circunvecinas representan verdaderos yacimientos de información sobre la cultura purépecha y sus antecesores, por lo que la caracterización del ambiente existente, tanto el inmediato antes del poblamiento del lugar, como el que prevaleció durante el desenvolvimiento de esta importante civilización, adquiere relevancia.

Los trabajos arquelógicos revelan que en la Cuenca de Zacapu, para la zona denominada "Las Lomas", existió una densa ocupación humana durante los primeros nueve siglos de nuestra era (Michelet, 1992; Arnauld et al., 1993; Arnauld et al., en prensa). Documentos históricos de la zona (Relación de Michoacán, 1977), indican que el paisaje actual es muy diferente al que conocieron las sociedades prehispánicas y colonial. Según los testimonios de los últimos purépechas, en la cienága se podía circular por canoas, y existían una serie de canales abiertos entre la densa vegetación acuática, misma que se explotaba para la elaboración de cestas y petates (Typha latifolia, Scirpus spp. y Cyperus spp.). Indudablemente la forma de vida de estos núcleos de población fue muy diferente a la actual, de lo cuál se desprenden algunas interrogantes sobre el paisaje: ¿se practicó agricultura en las orillas del lago y en "Las Lomas"?, ¿cómo fluctuó la orilla del lago a lo largo del tiempo?, ¿cuáles fueron los recursos naturales que pudieron utilizar los habitantes del lago?. Estas preguntas sólo pueden tener respuesta a través de estudios integrales de carácter interdisciplinario, dentro de los cuales el análisis palinológico suele constituir la columna vertebral.

\section{ZONA DE ESTUDIO}

El antiguo Lago de Zacapu se localiza en la porción norte del Estado de Michoacán, aproximadamente a $70 \mathrm{~km}$ al oeste de la ciudad capital Morelia, a una altitud promedio sobre el nivel del mar de $1975 \mathrm{~m}$ (fig. 1). Se trata de una llanura de origen palustre, de escasa pendiente y con ligeras elevaciones o domos de material silico-dolomítico-diatomítico, de no más de $10 \mathrm{~m}$ sobre el nivel de la actual ciénaga. Estas elevaciones que localmente reciben el nombre de "Lomas", constituyeron antiguas islas o penínsulas.

La litología de la cuenca está formada en su totalidad por material ígneo (Metcalfe y Harrison, 1984; Metcalfe, 1985). La joven planicie lacustre se encuentra circundada por montañas andesíticas y basálticas con elevaciones de poco más de 3250 msnm (Demant, 1981). La planicie central de la Cuenca es de rellenos aluviales-lacustrinos derivados de detritus ígneos, que han sufrido un proceso pedogenético muy marcado. En la mitad oeste de la cuenca abundan los derrames lávicos, superpuestos y escalonados con partes casi planas y con laderas de pendientes muy inclinadas. 

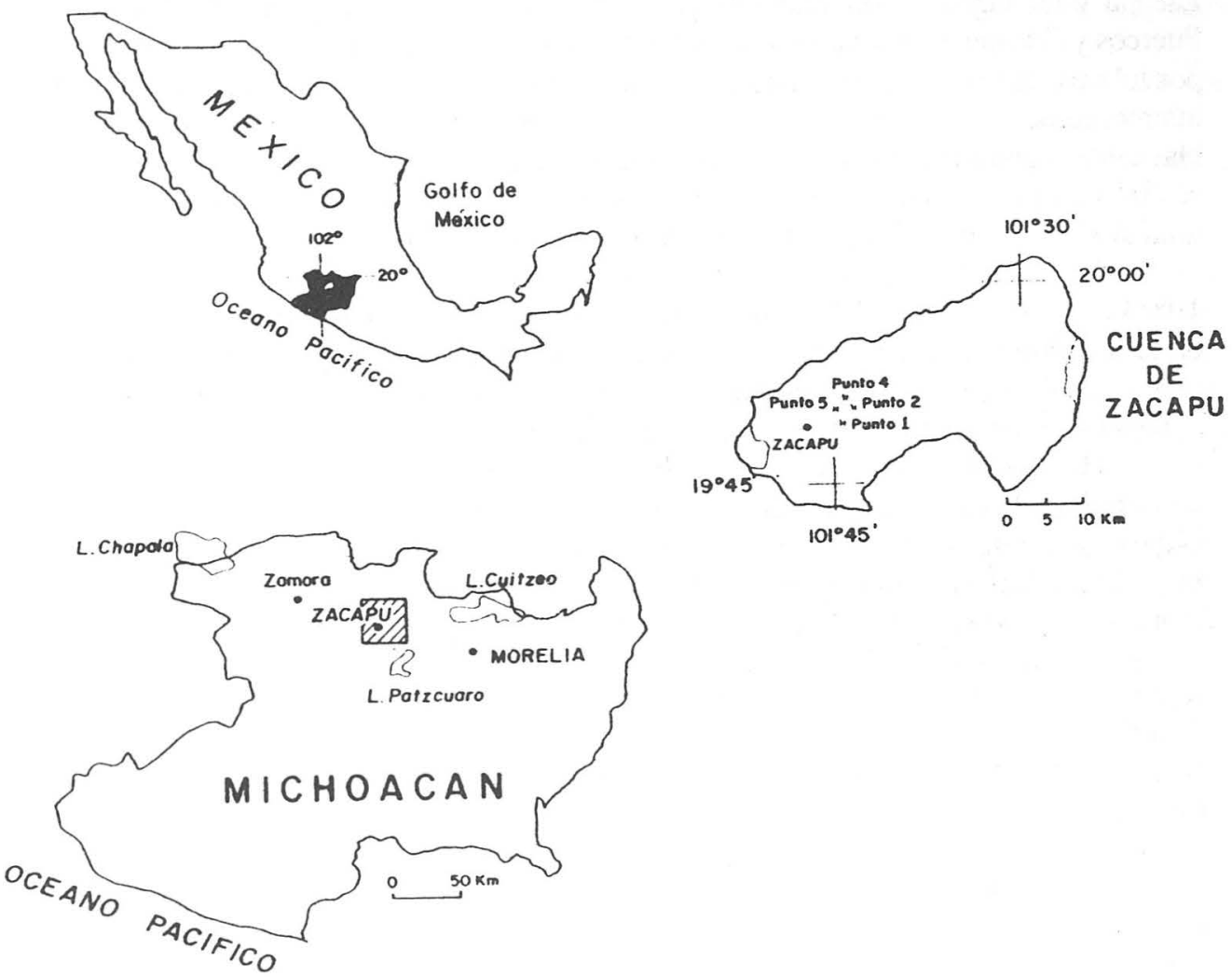

Fig. 1. Localización de la zona de estudio y de los sitios de toma de muestras. 
La Cuenca fue originalmente endorréica, generando un solo cuerpo de agua como lo sugiere la continuidad de la planicie lacustre, interrumpida sólo por las "penínsulas" e "islotes" ("Lomas"). Por un proceso de eutroficación y desecación natural, el lago original fue fragmentándose en varios cuerpos menores. Hacia la segunda mitad del siglo pasado el hidropaisaje lo constituía una amplia área pantanosa, la ciénaga de Zacapu y los lagos denominados García, Urumbécuaro, La Patera, La Zoromuta, Los Puercos y Zacapu. Los terrenos cenagosos entre los pequeños lagos estuvieron ocupados por tulares, carrizales de diversas asociaciones hidrofiticas y cruzados por canales que intercomunicaba la ciénaga. Hacia finales del siglo XIX, con el florecimiento de la Hacienda Cantabria, comenzó la desecación artificial de la ciénaga a través de un tajo que se abrió en una escotadura, en las inmediaciones del poblado Villa Jiménez. Las aguas drenan al Río Lerma (Michelet, 1992; Arnauld et al., 1993).

De acuerdo con la clasificación de Köppen modificada por García (García, 1988), las condiciones climáticas de una gran parte de la Cuenca pueden enmarcarse en la fórmula $\mathrm{Cb}(\mathrm{wl})(\mathrm{w})(\mathrm{e}) \mathrm{g}$, es decir, clima templado con verano fresco y largo, el más húmedo de los sub-húmedos; régimen de lluvias de verano con invierno seco, extremoso y con marcha térmica tipo Ganges.

Hoy en día, los terrenos de la ciénaga se destinan a la agricultura. Las comunidades vegetales que tienen influencia en la cuenca de captación del antiguo Lago de Zacapu son: bosque de encino-pino, bosque de encino, bosque de pino, bosque tropical caducifolio, matorral tropical, matorral espinoso, matorral rosetófilo, pastizal, izotal y vegetación hidrófila (Cruz y Xelhuantzi-López, en preparación).

Mediante el análisis palinológico se pretende: 1) conocer los elementos dominantes de la flora holocénica de la Cuenca del antiguo Lago de Zacapu, 2) asociar esta flora a comunidades vegetales, 3 ) inferir las condiciones climáticas del pasado, a través de las comunidades vegetales deducidas y 4) buscar elementos vegetales, agrícolas o asociados directamente con el hombre, que permitan conocer el uso que se dió a las "Lomas" y a la ribera del lago.

Con base en lo anterior, se buscan conclusiones generales respecto a: 1) la reconstrucción de las características paleoclimáticas y geodinámicas de la Cuenca del ex-lago de Zacapu y 2) los modos de uso agrícola de las márgenes del lago y de "Las Lomas".

\section{MATERIALES Y MÉTODOS}

La selección de los sitios para la toma y la obtención de muestras palinológicas se basó en el modelo etno-arqueológico desarrollado por Pierre Petrequin y Hervé Richard (Arnauld et al., en prensa), en cuencas lacustres de Francia y de Africa. Este 
modelo muestra que el estudio de la relación hombre/medio, en ambientes lacustres debe consistir en la correlación y comparación de varios sondeos, los cuales, además de proprcionar información a nivel regional, deberán incluir medios cercanos explotados por los habitantes del sitio:

De acuerdo con lo anterior, los puntos estudiados fueron los siguientes (fig. 1):

Punto 1. Ciénaga. Fondo del ex-lago, aproximadamente a $300 \mathrm{~m}$ de los sitios de ocupación y antiguos campos de cultivo.

Punto 2. Contacto orilla de las "Lomas"-ciénaga. Proximidad inmediata de los sitios de ocupación y campos de cultivo.

Punto 4. Pantano interno. En las "Lomas". Proximidad inmediata de los sitios de ocupación y campos de cultivo.

Punto 5. Loma Alta. En un sitio arqueológico de "Las Lomas".

No se cita el punto 3 porque no corresponde a muestras para análisis polínico.

Para la toma del material se utilizó una nucleadora rusa, especial para sedimentos blandos, prestada por el Laboratorio de Crono-Ecología de la Universidad de Besancon, Francia. También se recogieron muestras más superficiales, en una de las paredes de cada zanja seleccionada. Para la obtención de las muestras del punto 5, se aprovecharon las unidades de excavación arqueológicas, tomándose material tanto de sedimentos antrópicos como de sedimentos naturales.

Para la extracción de palinomorfos a partir de los sedimentos, el material se sometió al tratamiento con ácido fluorhídrico, clorhídrico y acetólisis de Erdtman (Erdtman, 1952). La observación y cuantificación de palinomorfos en las laminillas, se efectuó con microscopio fotónico, bajo el objetivo de inmersión (100X). Las preparaciones se encuentran depositadas en la Subdirección de Servicios Académicos del INAH y en el Instituto de Geología de la UNAM.

Por cada muestra obtenida, se estudió una laminilla. Se identificaron y contaron en su totalidad los granos de polen y esporas de helechos, algas, hongos presentes en cada una de las laminillas observadas. Palinomorfos de filiación incierta quedaron en la categoría de no identificados.

Se elaboraron gráficas o diagramas polínicos a partir de la relación porcentual de cada tipo polinico con respecto al total de palinomorfos.

\section{RESULTADOS}

\section{PINTOC I FONIOO DEL LA CIENAGA}

En el diagrama polínico obtenido para este sitio (fig. 2), se demarcan cinco etapas en el nivel del lago. En la primera (etapa I), comprendida entre $-3.85 \mathrm{~m}$ y -2.56 $\mathrm{m}$ (fechamiento por radiocarbono: $-3.55 \mathrm{~m}: 8110 \pm 115 ;-2.95 \mathrm{~m}: 7240 \pm 100$, años antes 


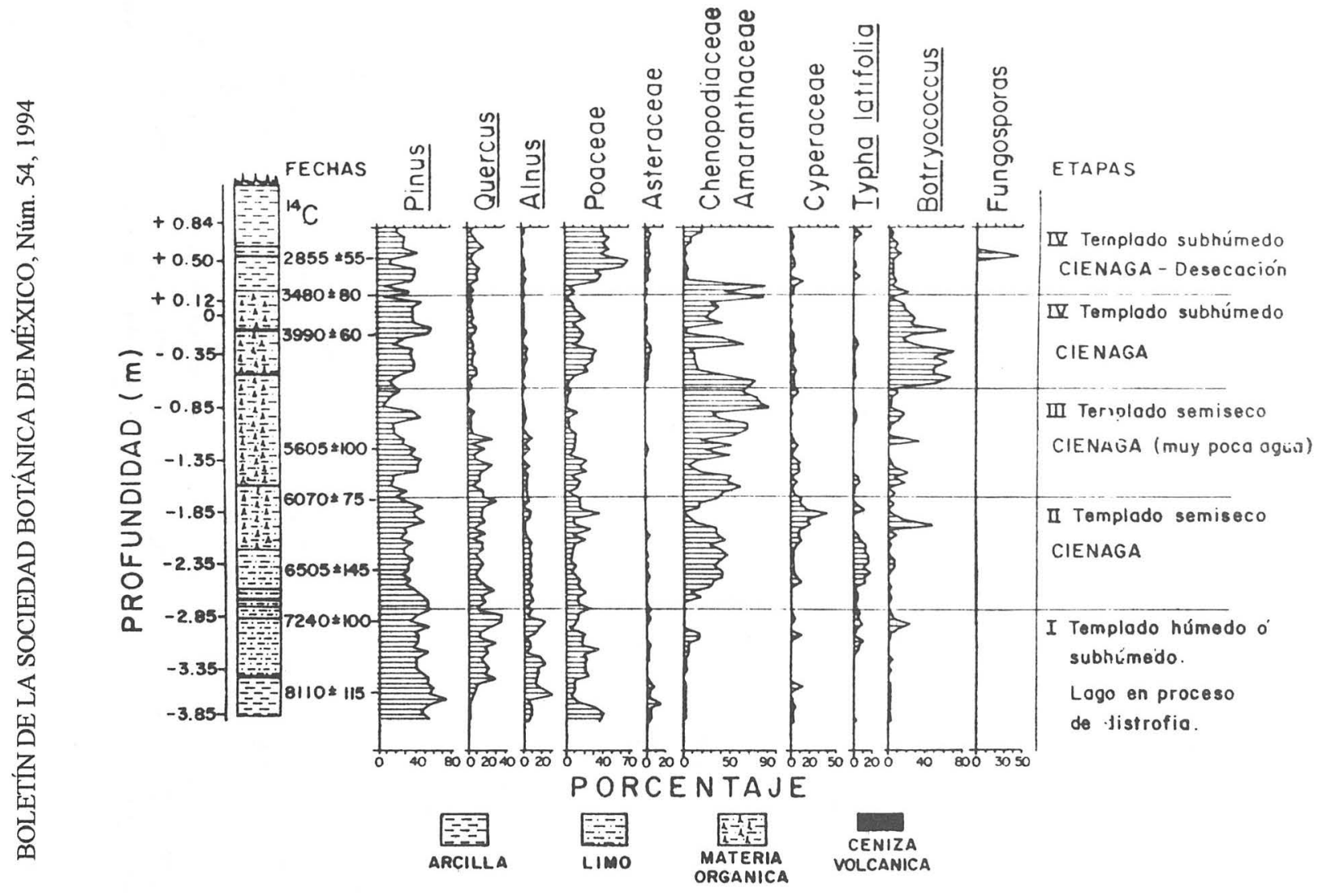

Fig. 2. Espectro palinológico del punto 1, fondo de la Ciénaga. 
del presente), el espectro palinológico indica que en los alrededores del antiguo lago de Zacapu, se desarrollaban bosques de pino-encino, con intercalaciones de algunos individuos de Alnus. En la actualidad, es común observar a las especies de Alnus formar parte de la composición florística natural de bosques asociados a ambientes húmedos o sub-húmedos generalmente templados. En esta primera etapa, los porcentajes de granos de polen de Alnus son relativamente altos. Los valores porcentuales de granos de Chenopodiaceae-Amaranthaceae y del polen de plantas acuáticas arraigadas son bajos, lo mismo que los del alga Botryococcus. Esto último sugiere que el agua del lago estaba bien oxigenada, aunque los estudios de diatomeas realizados por Metcalfe (Metcalfe, 1991; Metcalfe, en prensa), señalan algunas especies de aguas poco profundas, lo cual puede estar indicando el inicio del fenómeno de distrofia del lago y la existencia de zonas con diferentes profundidades y con desigualdades en el volumen de líquido, encontrándose áreas con un tirante de agua mayor y otras, tal vez las más numerosas, con características de pantano, todo ello favorecido por la topografia particular del sitio.

Las evidencias indican que desde principios del Holoceno, el de Zacapu no fué un lago hondo, aunque se piensa que fue durante esta etapa cuando alcanzó su profundidad máxima (Xelhuantzi-López, 1991). Basándose en el principio del actualismo, fundamental para la palinología y las reconstrucciones paleoambientales y aún con todos los inconvenientes que éste pueda tener, el tipo de vegetación inferido para los alrededores permite suponer para este tiempo, un clima templado húmedo o sub-húmedo. El porcentaje de granos de polen de árboles es alto, situación que denota la existencia en los alrededores de bosques densos y poco perturbados. Los porcentajes de Poaceae indican que también hubo comunidades de pastizales, seguramente primarios, tal y como ocurre en la actualidad (Cruz y Xelhuantzi-López, en preparación).

En la etapa II, de $-2.56 \mathrm{~m}$ a $-1.66 \mathrm{~m}$ (fechamiento por radiocarbono: $-2.35 \mathrm{~m}$ : $6505 \pm 145 ;-1.65 \mathrm{~m}: 6070 \pm 75$, años antes del presente), el porcentaje de polen de Alnus disminuye y se mantiene bajo. La gráfica del polen de Chenopodiaceae-Amaranthaceae señala valores altos y fluctuantes. El incremento de estas plantas comienza a ser notable precisamente por encima de las capas de ceniza volcánica. Las plantas acuáticas arraigadas se tornan importantes percibiéndose cierta sucesión en este tipo de plantas: primero es abundante Typha latifolia y después las Cyperaceae.

En este nivel, el espectro polínico sugiere que toda la zona se transformó en una ciénaga. Sobre las áres expuestas se favoreció el desarrollo de plantas oportunistas como las Chenopodiaceae-Amaranthaceae. El fenómeno de eutroficación del lago comienza a ser evidente por el incremento en las poblaciones de Botryococcus.

El decremento en las poblaciones de Alnus y la condición de ciénaga pueden ser indicio de una tendencia climática hacia condiciones más secas (clima templado semi-seco). 
En la etapa III, que se delimita de -1.66 a $-0.66 \mathrm{~m}$ (fechamiento por radiocarbono: $-1.15 \mathrm{~m}$ : $5605 \pm 100$, años antes del presente), los valores de Chenopodiaceae-Amaranthaceae muestran un fuerte incremento que posiblemente se debe a que el nivel de agua de la ciénaga disminuyó aún más y sobre los terrenos expuestos prosperaron un mayor número de poblaciones de estas plantas. Como hipótesis se propone que esta disminución en el nivel del agua obedeció al establecimiento de condiciones más secas (clima templado semi-seco).

En la etapa IV, artificialmente delimitada entre $-0.66 \mathrm{~m}$ y $+0.24 \mathrm{~m}$ (fechamiento por radiocarbono: $-0.14 \mathrm{~m}: 3990 \pm 60 ;+0.25 \mathrm{~m}: 3480 \pm 80$, años antes del presente), ocurre una ligera recuperación del polen de plantas arbóreas en conjunto (suma de pinos, encinos y ailes) y un notable incremento en los valores de Botryococcus. Lo anterior sugiere que en este período se elevó el nivel de agua de la cienága, inundándose los terrenos colonizados por las plantas oportunistas, lo que generó fenómenos de descomposición y putrefacción de la materia orgánica, aumentando la demanda de oxígeno del pantano. El carácter eutrófico del cuerpo de agua permitó el desarrollo de abundantes poblaciones de Botryococcus. Se puede suponer que el incremento de agua en la ciénaga y la recuperación de la vegetación arbórea fueron dadas por condiciones de mayor precipitación pluvial (clima templado sub-húmedo).

En la etapa V, demarcada de $+0.24 \mathrm{~m}$ a $+0.84 \mathrm{~m}$ (fechamiento por radiocarbono: $+0.55 \mathrm{~m}: 2855 \pm 55$ años antes del presente), con clara influencia humana manifestada por los restos arqueológicos -cerámica, utensilios y entierros- (Michelet, 1992; Arnauld et al., 1993), decrecen considerablemente los valores del alga Botryococcus y los de las Chenopodiaceae-Amaranthaceae, mientras que los de Poaceae aumentan. Estos cambios indican el reestablecimiento de la antigua ciénaga, que se conserva como tal, quizás con ligeras modificaciones en el nivel hídrico, hasta finales del siglo XIX cuando fue drenada artificialmente. La disminución del nivel del líquido en la ciénaga, en el paso de la etapa IV a la $\mathrm{V}$, no parece obedecer a cambios climáticos, sino más bien a fenómenos tectónicos y antrópicos, con la persistencia del clima templado sub-húmedo. Los estudios geológicos de la zona (Demant, 1992; Arnauld et al., 1993), indican que la actividad volcánica más importante ocurrió probablemente en el Holoceno. Por otro lado, los análisis de susceptibilidad magnética (Xelhuantzi-López y Urrutia, 1989; Xelhuantzi-López, en prensa) señalan altos valores de susceptibilidad en la porción superior del núcleo, que corresponden a una mayor concentración de minerales magnéticos o bien a una alta concentración aunada a cambios mineralógicos, mismos que se asocian con mayores tasas de erosión, principalmente de materiales pirocláticos. La sedimentología de las capas superiores (fig. 2), muestra varias tefras, evidencia inequívoca de eventos vulcanológicos. El fuerte aporte de sedimentos a la ciénaga pudo ser consecuencia de una disminución 
de las áreas boscosas tal y como lo sugiere la merma en los valores del porcentaje de polen de plantas arbóreas en conjunto. De acuerdo con las evidencias arqueológicas (Michelet, 1992; Arnauld et al., 1993), existe una importante ocupación de la zona desde hace poco más de 2000 años antes del presente, asentamientos que a través de sus prácticas agrícolas, de sus costumbres y ritos ceremoniales (Freddolino, 1973, señala que los antiguos purépechas ofrendaban imponentes incendios forestales al dios de los volcanes), contribuyeron a la deforestación y al incremento de la erosión.

No se encontró polen de plantas cultivadas.

\section{Punto 2 Contacto loma Cienaga}

El análisis palinológico del espectro del punto 2, permite distinguir tres etapas (figura 3). En la primera (etapa I), comprendida entre $-2.67 \mathrm{~m}$ y $-1.11 \mathrm{~m}$ (fechamiento por radiocarbono: $-1.77 \mathrm{~m}$ : $7610 \pm 150 ;-1.22 \mathrm{~m}$ : $6665 \pm 200$ años antes del presente), el espectro palinológico corrobora que en los alrededores del lago existieron bosques poco perturbados de pino-encino con intercalaciones de algunos individuos de Alnus. Al igual que hoy en día, en las inmediaciones de la zona también hubieron comunidades de pastizales tal como lo indican los altos valores porcentuales de Poaceae. El clima que predominó fue templado húmedo o sub-húmedo.

Los valores porcentuales de granos de polen de Asteraceae, Chenopodiaceae-Amaranthaceae, Cyperaceae, Typha latifolia, de fungosporas y la escasez de Botryococcus en esta etapa, sugieren un sitio con un suelo sobresaturado de agua, pero sin llegar a constituir un cuerpo de agua permanente. En los desplazamientos temporales de avance y retroceso de la orilla de la ciénaga, siempre quedó una zona fangosa.

En la etapa II, de $-1.11 \mathrm{~m}$ a $-0.50 \mathrm{~m}$ (fechamiento por radiocarbono: $-0.62 \mathrm{~m}$ : $5895 \pm 140$ años antes del presente), se percibe una disminución en el porcentaje del polen de plantas arbóreas y de gramíneas y un incremento considerable en el de Chenopodiaceae-Amaranthaceae junto con un pico de Asteraceae precisamente a $-0.50 \mathrm{~m}$. El registro de plantas hidrófilas es bajo y hay una ausencia prácticamente total de Botryococcus. Esta distribución de organismos sugiere el establecimiento de condiciones más secas que en la etapa I, como sería un clima templado semi-seco. En la ciénaga, las condiciones de menor humedad ocasionaron que el punto 2 , que pudo ser una área fangosa, se desecara casi por completo y que el pantano se retrajera.

La etapa III, delimitada de $-0.50 \mathrm{~m}$ a $+0.44 \mathrm{~m}$ (fechamiento por radiocarbono: $-0.18 \mathrm{~m}: 4470 \pm 75 ;+0.45 \mathrm{~m}: 2170+60$, años antes del presente), se caracteriza por un aumento en los valores porcentuales del polen de Pinus, Quercus, Poaceae y fungosporas aparejado con un decremento en los porcentajes de Chenopodiaceae-Amaranthaceae. 


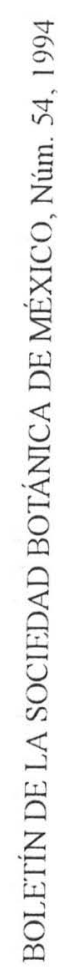

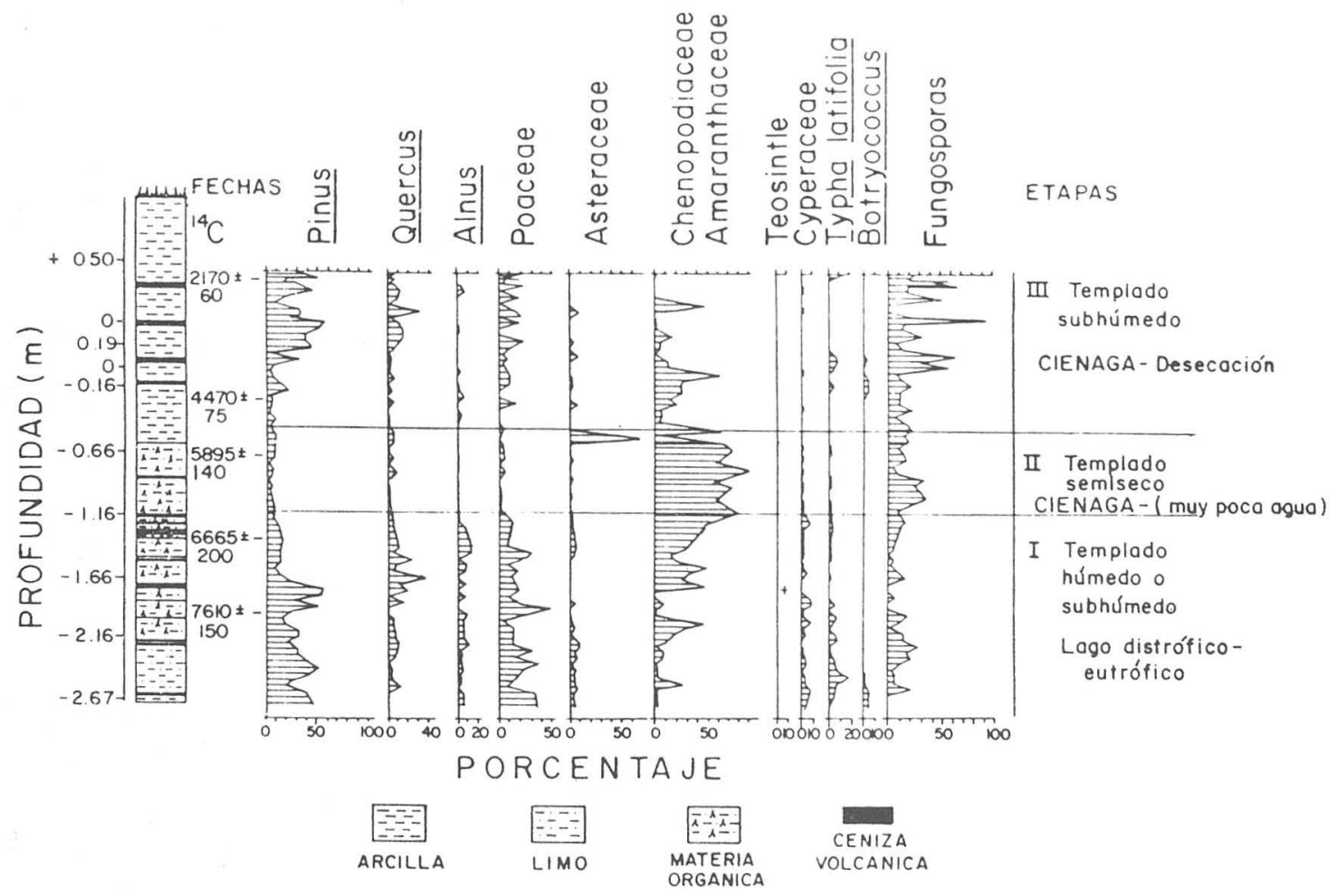

Fig. 3. Espectro palinológico del punto 2, contacto Loma-Ciénaga. 
El comportamiento de estas curvas sugiere aumento de humedad atmosférica y la existencia de un clima templado sub-húmedo, lo que permitió la recuperación de la vegetación boscosa de los alrededores, el re-establecimiento de la zona fangosa con la consecuente disminución de terrenos favorables para el desarrollo de plantas oportunistas y un incremento en la demanda de oxígeno.

El análisis polínico del punto 2 tampoco evidencia polen de plantas cultivadas o asociadas con cultivos; unicamente se encontró un grano de polen de Zea a $-1.76 \mathrm{~m}$, que por sus características morfológicas y por ubicarse en una época muy temprana (con más de 7500 años antes del presente), se identificó como teosintle.

\section{PINTO 4 PANTANO INTERNO}

El análisis palinológico del punto 4 (fig. 4) no sobrepasa los 3500 años antes del presente y se hizo con el fin de evaluar la eventual agricultura coetanea con la ocupación arqueológica de "Las Lomas". El espectro no pone de manifiesto polen de plantas cultivadas e indica el desarrollo de vegetación acuática arraigada principalmente de tipo tular. En el diagrama también se registra el polen de la vegetación regional, bosques de pino-encino con ailes entremezclados, aunque a excepción de los pinos, los porcentajes son más bien bajos, tal vez reflejando la presencia de bosques perturbados. El polen de Poaceae, Asteraceae y Chenopodiaceae-Amaranthaceae, familias que tienen representantes dentro de las malezas, es escaso.

Es importante hacer notar que la precisión del estudio en este sitio es menor que la de los puntos 1 y 2 ya que la separación entre cada una de las muestras fue de $10 \mathrm{~cm}$.

\section{PUNTO 5 LOMA ALTA}

El estudio palinológico de sedimentos en el sitio arqueológico de Loma Alta, se realizó con el propósito de descubrir granos de polen de plantas cultivadas, o en su defecto, de plantas ruderales que pudieran asociarse con prácticas agrícolas. Por lo que a granos de polen se refiere, en el sitio arqueológico de Loma Alta no se encontraron granos de polen, situación que impidió la obtención de un diagrama polínico. La causa de la ausencia de polen es la naturaleza del material que forma esta prominencia, que corresponde a sedimentos sílico-dolomítico-diatomítico, de granulometría gruesa que facilita que los palinomorfos se cuelen fácilmente. Con este resultado, es imposible aseverar o negar la existencia de verdaderos cultivos agrícolas. 


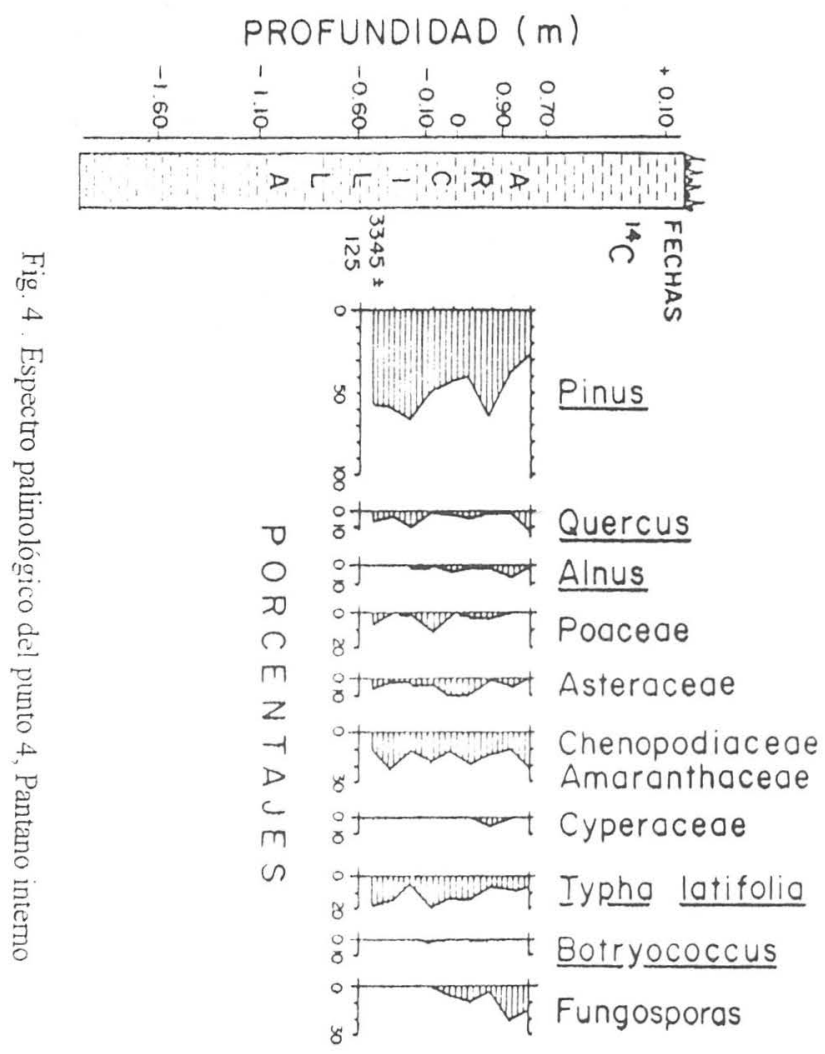




\section{CONCLUSIONES}

De los cuatro sitios estudiados, únicamente los puntos 1 y 2 aportan datos suficientes para lograr una interpretación paleoambiental a través del análisis polínico. La confrontación de los dos sitios señala diferencias en las etapas propuestas. En cuanto a fases climáticas, en ambos sitios se demarcan claramente tres: una temprana templada húmeda o sub-húmeda, de ca. 8100 a ca. 6700 años antes del presente; otra intermedia semi-seca, de ca. 6700 a ca. 5000 y la última templada subhúmeda, de ca. 5000 a la época actual.

A través del estudio se pone de manifiesto que las primeras modificaciones importantes de la vegetación regional ocurrieron hace poco más de 8000 años y se debieron a los efectos de erupciones volcánicas detectadas a través de los depósitos de cenizas (Arnauld et al., en prensa). En el lago, el nivel de agua era bajo, teniendo características de ciénaga. Entre 8000 y 7000 años antes del presente, ocurre un episodio tectónico severo (Arnauld et al., en prensa), detectado cualitativa y cuantitativamente en la sedimentación de los materiales (Xelhuantzi-López y Urrutia, 1989) y confirmado por los análisis polínicos, de susceptibilidad magnética (Xelhuantzi-López, en prensa) y de diatomeas (Metcalfe, 1991; Metcalfe, en prensa), al final del cual surgen "Las Lomas". Entre 6000 y 5000 años antes del presente, parece ocurrir el máximo descenso del nivel de agua de la ciénaga, índice de un clima más seco, episodio detectado también en las Cuencas de México y de Pátzcuaro (Brown, 1985: Watts y Bradbury, 1982).

El estudio sugiere que desde hace aproximadamente 7000 años antes del presente, el antiguo Lago de Zacapu ha sido un depósito de agua con características de ciénaga. Los cambios climáticos que pudieron inferirse a través del estudio palinológico, reflejan más variaciones en las condiciones de humedad que en las condiciones térmicas (parece que los últimos 7000 años antes del presente de la cuenca de Zacapu, han sido templados) y durante los últimos 5000 años antes del presente, el clima en el área de Zacapu, ha sido prácticamente el mismo que el actual, es decir, templado sub-húmedo.

Alrededor de 3000 años antes del presente, con el regreso de un clima un poco más húmedo, la ciénaga recupera extensión. Los bosques sufrieron fuertes desmontes y en el pantano la presencia del hombre es manifiesta por restos de cerámica y utensilios. No hay signos de implantación de agricultura en "Las Lomas".

En la modificación de la profundidad del lago, no puede considerarse como factor causal único al cambio de clima. El drenaje súbito por afallamiento provocado por tectonismo podría ser también motivo de la disminución en el nivel del lago. Desafortunadamente el estudio polínico no proporciona evidencias directas sobre este tipo de fenómenos sino únicamente sobre modificaciones en la flora. 
AgRADECIMIENTOS. Una versión modificada del análisis del núcleo 1. fue presentada por la autora, como tesis para obtener el grado de Maestro en Ciencias, investigación asesorada por los doctores Enrique Martínez Hernández y Socorro Lozano García, del Instituto de Geología de la UNAM. La autora manifiesta también su gratitud a las siguientes personas e instituciones que permitieron y apoyaron el dearrollo de este trabajo: del Centro de Estudios Mexicanos y Centroamericanos, a los doctores Dominique Michelet. Marie France Fauvet-Berthelot, Marie Charlotte Arnauld, Patricia Carot, Jean Noel Labat, Piere Petrequin. Herve Richard y Jean Meyer; del Instituto Nacional de Antropología e Historia a los compañeros de trabajo y a las autoridades.

De manera especial, se agradece al M. en C. Roberto Cruz Cisneros, de la Escuela Nacional de Ciencias Biológicas del Instituto Politécnico Nacional, sus sugerencias, comentarios y apoyo a la investigación.

\section{LITERATURA CITADA}

Arnauld, M.C., P. Carot y M.F. Fauvet-Berthelot. 1993. Arqueología de Las Lomas en la Cuenca Lacustre de Zacapu, Michoacán, México. Cuadernos de Estudios Michoacanos 5, CEMCA, México, D.F.

Arnauld, M.C., P. Carot, M.F Fauvet-Berthelot, R.L Rousseau, M. Magny. S. Metcalfe. P. Petrequin, O. Polaco, H. Richard. M.de los Rios y S. Xelhuantzi. 1994. La Cuenca de Zacapu en una secuencia de 8000 años. Evolución de los diferentes paisajes y primeros desmontes. Cuadernos de Estudios Michoacanos, CEMCA, México, D.F. (En prensa).

BROWN, R.B. 1985. A summary of Late-Quaternary pollen records from Mexico west of the Isthmus of Tehuantepec. Pollen Records of Late-Quaternary North American Sediments. V. N. Bryant and R.G. Holloway (Edrs.). American Association of Stratigraphic Palinologyst Foundations, Dallas. pp. 71-93.

DEMANT, A. 1981. L'axe néo-volcanique transmexicain. Etude volcanologique et pétrographique; signification géodynamique. Tesis de Doctorado de Estado. Universidad Aix-Marsella III.

Demant, A. 1992. Marco geológico regional de la Laguna de Zacapu, Michoacán. México. En: El Proyecto Michoacán 1983-1987. Cuadernos de Estudios Michoacanos 4. CEMCA, México, D.F.

ERdTMAN. G. 1952. Pollen morphology and plant taxonomy. Almquist and Wikesell. Stockolm.

Freddolino, M. K. 1973. An investigation into the "Pre-Tarascan" cultures of Zacapu. Michoacán, México. Ph. D. dissertation. Department of Anthropology. University of Yale.

GArcía, E. 1988. Modificaciones al sistema de clasificación climática de Köppen. Ed. E García. México, D.F. 
METCALFE, S. 1985. Late Quaternary environments of Central Mexico: a diatom record. Ph.

D. dissertation. School of Geography, Oxford.

Metcalfe, S. 1991. Changing environments of Zacapu Basin, Central Mexico: a diatom-based history spanning the last 30,000 years. School of Geography and Earth Resources No. 48, Oxford.

METCALFE, S. 1994. Estudio de las diatomeas de la Cuenca de Zacapu. Cuadernos de Estudios Michoacanos, CEMCA, México, D.F. México, D.F. (En prensa).

METCALFE, S. y S. P. HARrison. 1984. Cambio ambiental del Cuaternario Tardío en depósitos lacustres en la Cuenca de Zacapu, Michoacán. Reconstrucción preliminar. Bol. Inst. Geogr. 14:127-150.

Michelet, D.(Coord.). 1992. El Proyecto Michoacán 1983-1987. Cuadernos de Estudios Michoacanos 4, CEMCA, México, D.F.

RELACIÓN DE MICHOACÁN. 1977. Relación de las ceremonias y ritos de población y gobierno de los indios de la provincia de Michoacán (1541). Transcripción por José Tudela y estudio preliminar de José Corona Núñez, Balsal Editores, Morelia.

WatTs, W.A. y J.P. BradBury. 1982. Paleoecological studies at Lake Patzcuaro on the west-central mexican plateau and at Chalco in the Basin of Mexico. Quaternary Research 17(1): 56-70.

XELhuANTZi-López, M.S. 1991. Estudio palinológico y reconstrucción paleoambiental del ex-Lago de Zacapu, Michoacán. Tesis de Maestría, Facultad de Ciencias, UNAM, México, D.F.

Xeilhuantzi-López, M.S. 1994. Análisis de susceptibilidad magnética: punto 1-ciénaga. Cuadernos de Estudios Michoacanos, CEMCA, México, D.F. (En prensa).

Xel.huantzi-López, M.S. y J. UrRutia. 1989. Propiedades magnéticas de depósitos lacustres de Zacapu, Michoacán. Nueva herramienta en investigaciones arqueológicas. Trace 16. CEMCA. México, D.F. 